OPEN ACCESS

Edited by:

Robert Lindsay Medcalf, Monash University, Australia

Reviewed by: Stefania Ceruti, Università degli Studi di Milano, Italy Renee Jade Turner, University of Adelaide, Australia

*Correspondence: Yasuhiro Suzuk ya-suzuki@pha.ohu-u.ac.jp

Received: 29 September 2015 Accepted: 04 January 2016 Published: 25 January 2016

Citation: Suzuki Y, Nagai $N$ and Umemura $K$ (2016) A Review of the Mechanisms of Blood-Brain Barrier Permeability by Tissue-Type Plasminogen Activator Treatment for Cerebral Ischemia. Front. Cell. Neurosci. 10:2. doi: 10.3389/fncel.2016.00002

\section{A Review of the Mechanisms of Blood-Brain Barrier Permeability by Tissue-Type Plasminogen Activator Treatment for Cerebral Ischemia}

\author{
Yasuhiro Suzuki $^{1,2 *}$, Nobuo Nagai ${ }^{3}$ and Kazuo Umemura ${ }^{1}$ \\ ${ }^{1}$ Department of Pharmacology, Hamamatsu University School of Medicine, Hamamatsu, Japan, ${ }^{2}$ School of Pharmaceutical \\ Sciences, Ohu University, Koriyama, Japan, ${ }^{3}$ Faculty of Bioscience, Department of Animal Bioscience, Nagahama Institute of \\ Bio-Science and Technology, Nagahama, Japan
}

Cerebrovascular homeostasis is maintained by the blood-brain barrier (BBB), which forms a mechanical and functional barrier between systemic circulation and the central nervous system (CNS). In patients with ischemic stroke, the recombinant tissue-type plasminogen activator (rt-PA) is used to accelerate recanalization of the occluded vessels. However, rt-PA is associated with a risk of increasing intracranial bleeding (ICB). This effect is thought to be caused by the increase in cerebrovascular permeability though various factors such as ischemic reperfusion injury and the activation of matrix metalloproteinases (MMPs), but the detailed mechanisms are unknown. It was recently found that rt-PA treatment enhances BBB permeability not by disrupting the BBB, but by activating the vascular endothelial growth factor (VEGF) system. The VEGF regulates both the dissociation of endothelial cell (EC) junctions and endothelial endocytosis, and causes a subsequent increase in vessel permeability through the VEGF receptor-2 (VEGFR-2) activation in ECs. Here, we review the possibility that it-PA increases the penetration of toxic molecules derived from the bloodstream including rt-PA itself, without disrupting the BBB, and contributes to these detrimental processes in the cerebral parenchyma.

\footnotetext{
Keywords: brain ischemia, blood-brain barrier permeability, endothelial endocytosis, intracranial bleeding, tissuetype plasminogen activator, vascular endothelial growth factor
}

\section{INTRODUCTION}

The recombinant tissue-type plasminogen activator (rt-PA), a serine proteinase, is a thrombolytic agent that degrades fibrin clots through the activation of plasminogen to plasmin (Lijnen and Collen, 1987). Although rt-PA given within $3 \mathrm{~h}$ from the onset of ischemic stroke improves patients' clinical outcome, it induces a 10 -fold increase of symptomatic intracranial

Abbreviations: BBB, blood-brain barrier; BSA, bovine serum albumin; CNS, central nervous system; EC, endothelial cell; ECM, extracellular matrix; FITC, fluorescein isothiocyanate; HIF, hypoxia-inducible factor; ICB, intracranial bleeding; LRP, low-density lipoprotein receptor-related protein; MCA, middle cerebral artery; MMP, metalloproteinase; ROS, reactive radical oxide species; rt-PA, recombinant tissue-type plasminogen activator; VEGF, vascular endothelial growth factor; TJ, tight junction; $\mathrm{ZO}$, zonula occludens. 
hemorrhage (The National Institute of Neurological Disorders and Stroke rt-PA Stroke Study Group, 1995). Furthermore, rt-PA treatment delayed beyond $3 \mathrm{~h}$ is associated with an increased risk of hemorrhagic transformation with enhanced brain injury (Clark et al., 1999). Subsequently, the European Cooperative Acute Stroke Study showed that rt-PA administered between 3 and $4.5 \mathrm{~h}$ after the onset of symptoms significantly improved the clinical outcomes of patients with acute ischemic stroke, but it increased the risk of symptomatic intracranial hemorrhage. It was confirmed that delayed treatment beyond $4.5 \mathrm{~h}$ was not associated with a statistically significant benefit (Hacke et al., 2008). A number of clinical studies using magnetic resonance imaging provided evidence that rt-PA treatment is associated with blood-brain barrier (BBB) breakdown (Kastrup et al., 2008; Kassner et al., 2009), which also correlates with an increased risk of hemorrhagic transformation during thrombolysis in ischemic stroke (Kassner et al., 2009). These findings strongly suggest a causal relationship between rt-PA and BBB breakdown in the ischemic human brain.

Although the deleterious effect of rt-PA after ischemic stroke has been widely accepted, it remains unclear whether many blood-derived rt-PAs penetrate the brain and contribute to these detrimental processes in the cerebral parenchyma. Intravenously administered rt-PA has been shown to cross brain endothelial cells (ECs) via two ways: (1) by binding to the surface; and (2) transcytosis without compromising the BBB integrity (Benchenane et al., 2005; López-Atalaya et al., 2007). rt-PA was also found to enter the parenchyma under pathological conditions where it further affects BBB breakdown ( $\mathrm{Su}$ et al., 2008). Some hypotheses have been proposed to explain how rt-PA within the parenchyma exacerbates intracranial bleeding (ICB) after ischemic stroke. Extracellular rt-PA can mediate some of its actions either through plasmin; the degradation of laminin, one of the extracellular matrix (ECM) proteins (Chen and Strickland, 1997); the activation of microglia (Rogove and Tsirka, 1998); or the excessive induction of vascular remodeling and angiogenesis via overactivation of metalloproteinases (MMPs; Suzuki et al., 2007; Yamashita et al., 2009; Won et al., 2014) and the vascular endothelial growth factor (VEGF; Kanazawa et al., 2011; Suzuki et al., 2015). There is also evidence that rt-PA may have a direct toxic effect on the ischemic brain (Wang et al., 1998; Nagai et al., 1999), possibly through activation of the N-methylD-aspartate receptor (NMDAR; Nicole et al., 2001). This may be of particular importance given that rt-PA diffused within the cerebral parenchyma after ischemia can have detrimental effects including enhancing the neurotoxic processes (Kaur et al., 2004).

In the present review, we firstly described $\mathrm{BBB}$ breakdown by rt-PA, and then discussed the role of the enhancement of $\mathrm{BBB}$ permeability without compromising $\mathrm{BBB}$ integrity on $\mathrm{BBB}$ breakdown. Especially, we focus on the involvement of VEGF in ECs as the first step in BBB breakdown by the deleterious effect of rt-PA after ischemic stroke. In addition, it is described the possibility that an inhibition the enhancement of $\mathrm{BBB}$ permeability without compromising $\mathrm{BBB}$ integrity may extend the therapeutic time widow by rt-PA for ischemic stroke.

\section{BBB AND ENDOTHELIAL TIGHT JUNCTIONS}

The BBB is formed by endothelial tight junctions (TJs) together with pericytes, perivascular astrocytes, and basement membrane in the vasculature. Furthermore, as cerebrovascular function is regulated by the neuronal environment, the $\mathrm{BBB}$ and neurons form a functional unit called the neurovascular unit. TJs are constituted by multiple protein components that involve transmembrane proteins (e.g., occludin, claudins, and junctionassociated molecules) linked to the actin cytoskeleton via cytoplasmic zonula occludens ( $\mathrm{ZO}$ ) proteins. Transmembrane proteins, occludin and claudins are critical for paracellular function at the BBB (Hawkins and Davis, 2005). Claudins are small transmembrane proteins $(20-24 \mathrm{kDa})$ that span the membrane four times; claudin-1, -3 , and -5 are expressed in ECs of the BBB. Occludin is a $60-65 \mathrm{kDa}$ phosphoprotein highly expressed in cerebral endothelia, but it is sparsely distributed in peripheral endothelia (Hirase et al., 1997). The overexpression of claudins can induce the formation of TJs, but the expression of occludin does not lead to the formation of TJs. Thus, it is likely that claudins form the primary seal of TJs, and occludin acts as an additional support structure. Claudin and occludin are anchored to the actin cytoskeleton via ZO-1 (Hawkins and Davis, 2005).

\section{rt-PA and Reperfusion Injury}

In patients with ischemic stroke, rt-PA increases the risk of ICB via $\mathrm{BBB}$ breakdown through a number of mechanisms. One of these mechanisms is thought to be by reperfusion after the degradation of occlusive blood clots by rt-PA. Brain parenchymal damage occurs because of a complex series of events in the setting of ischemia followed by reperfusion injury. These events start due to an interruption in blood flow to the affected tissue followed by the depletion of cellular energy resources and glycolysis at an anerobic substrate level, with subsequent lactic acidosis, failure of the sodium potassium pump, the release of glutamate, cytotoxic edema, and free radical formation (Nour et al., 2013). The activation of both innate and adaptive immune responses also creates free radicals. This excessive generation of free radicals overwhelms the system, which then becomes inefficient in scavenging these molecules, leading to BBB breakdown. Furthermore, ICB associated with ischemic infarction is recognized due to ischemia followed by reperfusion, and both the rate of ICB and stroke outcome can be increased by the duration of reperfusion from the onset of vessel occlusion due to ischemic stroke (Jickling et al., 2014). Additional injury is extensively shown in the brain and in other tissues, which is mediated by reactive radical oxide species (ROS). ROS contribute to $\mathrm{BBB}$ disruption by several mechanisms: oxidative damage to cellular molecules (i.e., proteins, lipids, and DNA); the activation of MMPs and subsequent degradation of basement membrane; cytoskeletal reorganization of ECs; the modulation of TJ proteins and upregulation of inflammatory mediators; and subsequent additional and extensive reperfusion injury (Kahles and Brandes, 2012). 


\section{rt-PA Promotes ICB Through Mechanisms Beyond its Role in Thrombolysis and Reperfusion}

rt-PA increases BBB permeability via degradations of basement membrane and TJ proteins. These degradations are associated with plasmin activation, low density lipoprotein receptor associated protein-1 (LRP-1) stimulation, and MMPs induction (Yamashita et al., 2009; Won et al., 2014). As a result, rt-PA exacerbates ischemic brain damage and ICB by increasing BBB permeability.

Plasmin, which is activated by rt-PA, can directly degrade fibrin clots and basement membrane components such as collagen IV (Mackay et al., 1990; Lukic-Panin et al., 2010), laminin (Chen and Strickland, 1997) and fibronectin (Marchina and Barlati, 1996), or via the activation of MMPs (Lijnen, 2001), which possess similar basement membrane dismantling capabilities and damages the TJs (Jin et al., 2010).

LRP is one of the major binding sites of rt-PA (Bu et al., 1992) on the cell surface. LRP, a member of the lipoprotein receptor family, is a scavenger receptor that binds a variety of biological ligands associated with the ECM and is thought to be primarily involved in lipoprotein metabolism (Herz et al., 1988), and in the clearance of protease-inhibitor complexes in the adult brain (Bu et al., 1992). Furthermore, the increase in $\mathrm{BBB}$ permeability by rt-PA occurs via the activation of LRP (Yepes et al., 2003; Benchenane et al., 2005; Su et al., 2008; Suzuki et al., 2009; Niego and Medcalf, 2014). LRP is selectively upregulated in ECs under ischemic stress, and LRP activation by binding to rt-PA stimulates signal pathways such as the nuclear factor $\kappa \mathrm{B}$ pathway (Suzuki et al., 2009).

MMPs, a family of zinc endopeptidases, contribute to tissue remodeling through the degradation of ECM proteins. Although clarifying the precise timing and release of cells to MMP after ischemia requires further study, MMPs, including MMP-9 (gelatinase B), MMP-2 (gelatinase A), and MMP-3 (stromelysin-1), are thought to be key molecules involved in BBB opening and ICB after ischemic stroke (Rosenberg et al., 1995; Castellanos et al., 2003; Wang et al., 2003; Suzuki et al., 2007; Mishiro et al., 2012; Jickling et al., 2014) together with other brain proteases (i.e., plasmin, endogenous t-PA, and urokinase; Wang and Shuaib, 2007). Claudin-5 and occludin, components of the TJs, contain extracellular MMP cleavage sites and are a direct substrate of MMPs (Wachtel et al., 1999; Yang et al., 2007), suggesting that MMPs can degrade TJs directly. As the structural disruption of the interaction between occludins and actin filaments can lead to the perturbation of paracellular permeability (Madara et al., 1986), the degradation of occludin by MMPs is likely to trigger $\mathrm{BBB}$ opening. MMPs also degrade components of basement membrane and contribute to BBB impairment, vasogenic edema and hemorrhagic transformation (Rosell et al., 2008). MMP-9 especially is believed to play a major role in $\mathrm{BBB}$ disruption during ischemic stroke because ischemic stress induces MMP-9 and the plasma MMP-9 concentration, which strongly correlate with patients' stroke severity (Horstmann et al., 2003; Jin et al., 2010; Reuter et al., 2013).

MMP-3 is also thought to be involved in ICB due to delayed rt-PA treatment. MMP-3 is produced by pericytes (Yang et al., 2013) and ECs (Suzuki et al., 2007) after ischemic stroke. The increase in ICB caused by the delayed rt-PA treatment was impaired in mice with a gene deficiency of MMP-3, and a broad spectrum MMP-inhibitor suppressed ICB in wildtype mice but not in MMP-3 deficient mice (Suzuki et al., 2007). MMP-3 can be activated by plasmin (Lijnen, 2001), and it has a broad-spectrum substrate specificity, including pro-MMP-9, which is activated by limited cleavage (Nagase and Woessner, 1999). Furthermore, rt-PA treatment induced MMP-3 selectively in ECs at the ischemic damaged area in a mouse stroke model (Suzuki et al., 2007), and MMP-3 was increased in a postmortem human stroke brain (Jickling et al., 2014). These findings indicate that MMP-3 is also involved in degrading the barrier of blood vessels and contributing to ICB.

\section{Increase in BBB Permeability by Paracellular Transport and Transcytosis}

rt-PA treatment is thought to enhance ICB via the acceleration of $\mathrm{BBB}$ disruption after ischemic stroke. However, there is still a possibility that rt-PA treatment enhances $\mathrm{BBB}$ permeability without $\mathrm{BBB}$ disruption after ischemic stroke.

The BBB is composed of blood vessels whose ECs display extremely low rates of transcellular vesicular transport (transcytosis) due to pinocytic activity (Reese and Karnovsky, 1967; Pun et al., 2009; Saunders et al., 2012; Siegenthaler et al., 2013). In concert with pericytes and astrocytes, this unique brain endothelial barrier seals the central nervous system (CNS) and controls substance influx and efflux (Armulik et al., 2010; Bell et al., 2010; Daneman et al., 2010). BBB permeability is regulated in response to various stimulators or stressors, which can exert beneficial or deleterious effects on the brain depending on the context, timing, and functional cellular outcomes of signaling (Roux and Couraud, 2005). BBB permeability can be increased via two processes. The first is paracellular transport, which is associated with loosening the TJs between ECs. The expression of occludin, a component of TJs, is highly suppressed by a number of pathological stresses, including oxidative stress, and a decrease in the occludin expression results in the increase in BBB permeability (Ramirez et al., 2009; Lochhead et al., 2010). To enhance paracellular transport, rt-PA seems to decrease occludin through ROS generation associated with reperfusion and/or through the activation of MMPs.

The second process is transcytosis, which is consistent with endocytosis and involves vesicle transport to the opposite side of the cell and exocytosis. Peripheral ECs display active vesicle trafficking to deliver nutrients to peripheral tissues, whereas CNS ECs express transporters to selectively traffic nutrients across the BBB (Saunders et al., 2012; Siegenthaler et al., 2013). However, it is still unclear when and how ECs are transported by transcytosis. 


\section{VEGF}

VEGF stimulates endocytosis and transcytosis (Horowitz and Seerapu, 2012; Nakayama and Berger, 2013). VEGF binds to two receptor-coupled protein tyrosine kinases (Tyr), VEGF receptor 1 (VEGFR-1, Flt-1) and VEGFR-2 (i.e., fetal liver kinase 1 [Flk-1] or the kinase insert domain receptor). VEGF regulates the dissociation of TJs under ischemic condition (Fischer et al., 2002; Figure 1) and endocytosis (Horowitz and Seerapu, 2012), and the subsequent increase in vessel permeability through VEGFR-2 activation, which contributes to cerebral swelling at the early stage after ischemic stroke (Abumiya et al., 2005). VEGF also has a fundamental role in vascular remodeling and angiogenesis by increasing EC proliferation, migration, and microvascular hyperpermeability (Brown et al., 1992). The downstream elements of VEGFR-2 signaling include the Ras/Raf/MEK pathway, which leads to EC proliferation; PI3K-AKT/PKB pathway, which supports EC survival; and p38/MAPK-HSP27 pathway, which promotes EC migration (Obermeier et al., 2013). Vascular remodeling is an important component of recovery after stroke, although it makes vessels to be leakier and prone to intracranial hemorrhage (Durukan et al., 2009). Thus, they may also promote intracranial hemorrhage.

VEGF is expressed in the normal adult brain, mainly in epithelial cells of the choroid plexus, as well as in astrocytes and neurons, such as granule cells of the cerebellum (Monacci et al., 1993; Marti and Risau, 1998). VEGF expression is regulated by extensive signaling pathways. Among them, hypoxia is a strong inducer of VEGF messenger (m)-RNA expression in many cells in vitro and in vivo (Banai et al., 1994; Ikeda et al., 1995; Kovács et al., 1996; Hayashi et al., 1997). The two transcription factors, hypoxia-inducible factor-1 (HIF-1) and

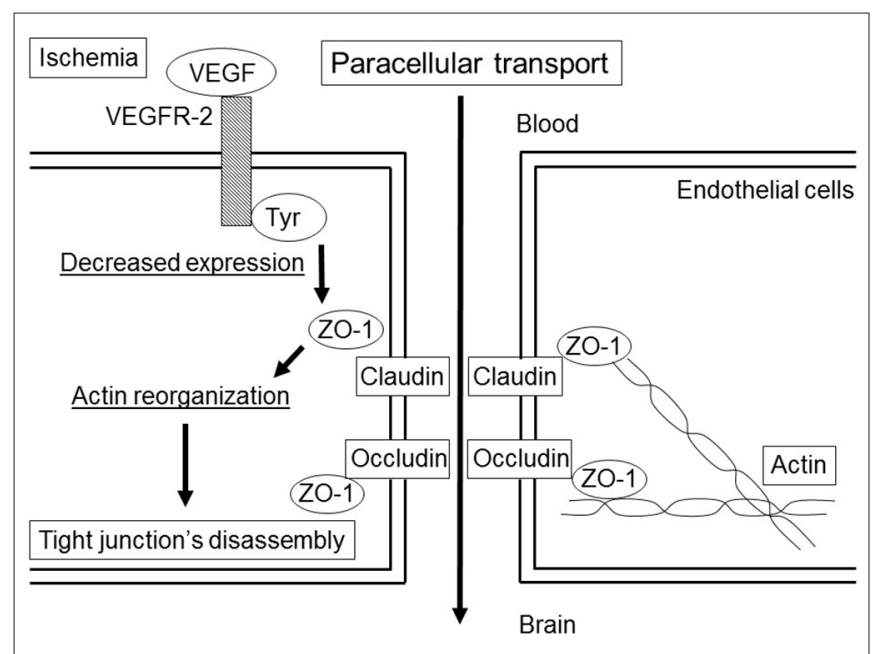

FIGURE 1 | Paracellular transport in the blood-brain barrier (BBB) by the vascular endothelial growth factor (VEGF) under ischemic condition. The binding of VEGF to the VEGF receptor-2 (VEGFR-2) induces the activation of tyrosine kinase (Tyr), which leads to a decrease in the expression of zonula occludens (ZO)-1. The changes in ZO-1 correlate with small changes in action distribution, which induce disassembly of the tight junction (TJ).
HIF-2 are involved in the regulation of VEGF expression. HIF-1 is composed of a hypoxia-regulated $\alpha$-subunit and a $\beta$-subunit, and is a basic helix-loop-helix heterodimeric transcription factor activated by reduced oxygen tension (Wenger and Gassmann, 1997). HIF- $1 \alpha$ is continuously produced and rapidly degraded under normoxia (Sharp and Bernaudin, 2004), whereas it is degraded slowly under hypoxia, and allowed rapid accumulation and binding to hypoxia-responsive elements (Shi, 2009). HIF-2, a homolog of HIF-1, is also involved in the regulation of VEGF gene expression (Ema et al., 1997). HIF-2 has an additional role in the regulation of VEGFR-2 (Kappel et al., 1999). Although VEGF is induced in ECs through HIF-1 $\alpha$ (Tang et al., 2004), and the expression of HIF-1 and HIF-2 are increased in the border area of ischemic stroke (Marti et al., 2000), the mechanisms by which VEGF gene expression is regulated during cerebral ischemia remain unclear.

One mechanism for the increase in VEGF secretion by cells exposed to ischemia is an increase in its transcription rate mediated by the binding of HIF-1 to a hypoxia-responsive element in the $5^{\prime}$-flanking region of the VEGF gene, as is observed in PC12 cells (Levy et al., 1995), bovine pulmonary artery ECs (Liu et al., 1995), and Hep3B cells (Forsythe et al., 1996). Other mechanisms include the increase in VEGF mRNA stability (Ikeda et al., 1995) and efficient translation of VEGF mRNA through an internal ribosome entry site (Stein et al., 1998).

\section{rt-PA, HIF-1, and VEGF}

rt-PA increases VEGF expression in bone marrow-derived myeloid cells, cultured cerebral cortical neurons, and ECs (Ohki et al., 2010; Wu et al., 2012; Duan and Ni, 2014; Suzuki et al., 2015). In addition, rt-PA affects HIF- $1 \alpha$ regulation. rt-PA induces HIF- $1 \alpha$ accumulation in the ischemic brain and accelerates HIF- $1 \alpha$ accumulation mediated by the mammalian target of rapamycin in cultured neurons (Wu et al., 2012). rt-PA treatment after ischemia does not enhance the expression of VEGF through the nuclear accumulation of HIF-1 $\alpha$ in a transformed mouse brain EC line, bEnd.3 (Suzuki et al., 2015); instead, the overexpression of t-PA stimulates VEGF expression in ECV304, a human immortalized EC due to the stimulation of ERK/p38 signaling pathways (Duan and $\mathrm{Ni}$, 2014). These findings suggest a possibility that rt-PA accelerates the expression of VEGF via HIF- $1 \alpha$ or the signal pathway for the upregulation of HIF- $1 \alpha$ in parallel without VEGF induction.

The inhibition of VEGF signaling reduces rt-PA-related ICB (Kanazawa et al., 2011; Suzuki et al., 2015), suggesting that VEGF has a deleterious role in ICB. Similarly, VEGF administered within 1-24 $\mathrm{h}$ from stroke onset increases in the rate of $\mathrm{BBB}$ breakdown and hemorrhagic transformation, and the size of infarction in rodents (Zhang et al., 2000; Abumiya et al., 2005). Taken together that VEGF increases microvascular permeability to blood plasma proteins within minutes after its administration (Dvorak et al., 1995), the induction of VEGF after ischemic stroke may enhance the detrimental effects. In contrast, VEGF administered $48 \mathrm{~h}$ from 
stroke onset enhances angiogenesis and improves neurologic recovery, and improves cerebral blood flow 28 days after stroke (Zhang et al., 2000). The effect of VEGF on angiogenesis is longer than on permeability. Newly formed vessels in ischemic mouse brains are first visible within 4 days (Dellian et al., 1996) and there is an increase in newly formed vessels in ischemic mouse brains 10 days after VEGF treatment but not in contralateral non-ischemic brains (Zechariah et al., 2013). Thus, VEGF seems to have biphasic roles in stroke, it promotes $\mathrm{BBB}$ breakdown and hemorrhagic transformation in the early stage, within $24 \mathrm{~h}$, but promotes $\mathrm{BBB}$ integrity and vascular function in the late stage, over $48 \mathrm{~h}$, after ischemic stroke.

\section{Paracellular Permeability by rt-PA}

Increased paracellular permeability is correlated with the disruption of TJs (Kevil et al., 2000; Mark and Davis, 2002; Lee et al., 2004). Until now, however, the role of TJs in vascular permeability of either plasma components or circulating cells was supported by the results of only a few studies (Martìn-Padura et al., 1998; Pedram et al., 2002). During ischemic stroke, temporal hypoxemia for $10 \mathrm{~min}$ increased $\mathrm{BBB}$ permeability associated with alterations in $\mathrm{TJ}$ protein expression (Witt et al., 2003). Accordingly, immunoreactivity of ZO-2 or claudin-5 was significantly reduced in infarct regions compared with non-infarct regions $24 \mathrm{~h}$ after ischemia (Fischer et al., 2007). However, according to ultrastructural analyses at 5 and $25 \mathrm{~h}$ after ischemia, fluorescein isothiocyanate (FITC)-albumin was extravased around vessels with intact TJs, whereas the endothelium exhibited an enhanced transcellular vesicle trafficking (Krueger et al., 2013). Additionally, the morphology of TJ components identified by antibodies against occludin and claudin-5 appears to be regularly maintained in regions where FITC-albumin massively leaked into the neuropil $25 \mathrm{~h}$ after ischemia (Krueger et al., 2013). A conclusive time frame for TJ reassembly following the disruption of ischemia is currently lacking. In Madin-Darby canine kidney cells, permeability is returned to the same levels of the initial condition $5 \mathrm{~h}$ after ATP repletion. After ATP depletion for $1 \mathrm{~h}$ and repletion for $3 \mathrm{~h}$, occludin was once again found almost exclusively at the level of the TJs. This reversible shift is inhibited by the chelation of intracellular calcium. In contrast, ZO-1 is not significantly altered during ATP depletion or repletion (Ye et al., 1999). However, VEGF specifically down-regulates claudin-5, occludin protein, and mRNA. In the mouse cerebral cortex, the microinjection of VEGF disrupted claudin-5 and occludin, and induced loss of barrier function (Argaw et al., 2009). The continuity of the ZO-1 expression was significantly disrupted during $1.5 \mathrm{~h}$ of hypoxia in primary cultures isolated from porcine brain ECs. Furthermore, VEGF alone or $\alpha$-lipoic acid alone did not change ZO-1 localization in the primary culture, however, VEGF in combination with $\alpha$-lipoic acid decreased the ZO-1 expression to nearly the same extent as $3 \mathrm{~h}$ of hypoxia (Fischer et al., 2002). These results suggest that ischemia increases the paracellular flux via the release of VEGF, which in turn leads to the dislocalization, decreased expression, and enhanced phosphorylation of TJs. As TJ proteins are responsible for the paracellular permeability across the BBB, VEGF induced by ischemia may increase paracellular permeability of $\mathrm{BBB}$ via suppression of the expression of TJ proteins in the early stage of ischemic stroke (Fischer et al., 2002; Argaw et al., 2009, 2012). However, it is possible that BBB permeability is increased by TJs loosening in the early stage of ischemic stroke because a study found intact TJs at $24 \mathrm{~h}$ after stroke (Krueger et al., 2013).

Delayed rt-PA treatment enhances the fragmentation of occludin and claudin-5 $24 \mathrm{~h}$ after middle cerebral artery (MCA) occlusion in rats (Won et al., 2014), and reduces claudin-5 at $24 \mathrm{~h}$ (Ishiguro et al., 2010) or occludin and ZO-1 at $48 \mathrm{~h}$ after ischemia in mice (Mishiro et al., 2012). Furthermore, it was found that blood levels of TJ proteins were higher in patients with hemorrhagic transformation than in those without hemorrhagic transformation (Kazmierski et al., 2012). These findings indicate that delayed rt-PA treatment results in the enhancement of ICB due to stroke by the loss of TJ proteins at a relatively later stage, over $24 \mathrm{~h}$, on ischemic stroke. However, the role of TJ proteins in early ICB is still unclear. Delayed rt-PA treatment may enhance the $\mathrm{BBB}$ permeability through the paracellular pathway by the degradation of TJ proteins without $\mathrm{BBB}$ breakdown as well as ischemia without rt-PA treatment.

\section{rt-PA Treatment and Endocytosis}

The intravenous treatment of rt-PA does not increase either albumin extravasation or ICB in naive mice (Cheng et al., 2006; Su et al., 2008; Suzuki et al., 2015). Additionally, the intraventricular injection of rt-PA does not increase Evans blue extravasation in sham-operated mice (Yepes et al., 2003). In contrast, an intravenous injection of rt-PA slightly increases the Evans blue extravasation in native mice (Turner and Vink, 2012). Furthermore, an intravenous injection of biotinylated rt-PA with fluorescent dextran $(77 \mathrm{kDa})$ is detected the extravasation of rt-PA in the brain parenchyma of nonlesioned animals (Benchenane et al., 2005), suggesting that rt-PA itself and other plasma molecules can cross the intact $\mathrm{BBB}$ via transcytosis. Similarly, we observed that delayed rt-PA treatment dramatically increased endocytosis of cerebral ECs at the ischemic border region together with an increase in the existence of gold-labeled bovine serum albumin (BSA) administered intravenously at the vascular lumen, inside ECs, in the basement layer of the ECM and extravascular space without obvious TJ defects in mice (Suzuki et al., 2015). This indicates that delayed rt-PA treatment increased extravasation of BSA at the ischemic border region in the early period after MCA occlusion by the additional acceleration of transcytosis rather than by the degradation of vascular structures. It is likely that the administration of rt-PA accelerates extravasation of phagocytic vesicles, including rt-PA, and interacting plasma plasminogen in the parenchyma by the upregulation of transcytosis increases the likelihood of a plasmindependent BBB alteration at the perivascular space. Because this study does not provide direct evidence for the involvement of exocytosis, another component of the transcytosis process, in the ischemic border region of the extravasation of BSA, there is still 
the possibility that endothelial endocytosis is independent of $\mathrm{BBB}$ opening. Additionally, transcytosis may possibly be involved in the increase in $\mathrm{BBB}$ permeability by the delayed treatment of rt-PA after ischemic stroke (Figure 2).

\section{Endocytosis and the Multifunction of LRP}

As previously described, LRP is a scavenger receptor that binds a variety of biological ligands associated with the ECM, and it is a major binding protein of rt-PA (Bu et al., 1992). LRP acts as a membrane receptor of rt-PA, and its activation induces the expressions of MMPs and VEGF (Wang et al., 2003; Suzuki et al., 2009, 2015). The invasion of carcinoma cells is also decreased by LRP silencing with RNA interference despite a strong stimulation of pericellular MMP-2 and urokinase-type plasminogen activator proteolytic activities (Dedieu et al., 2008). However, the precise role of LRP in the regulation of ECM remodeling is still unclear.

During endocytosis, LRP does not act alone, as it has membrane partners that vary according to numerous parameters, including the cell origin, ECM composition, and pathological conditions (Etique et al., 2013). LRP-mediated endocytosis of soluble ligands is usually followed by intracellular lysosomal routing and catabolism. LRP emerges as an endocytic receptor regulating cellular matrix attachment sites and coordinating the balance of adhesion/deadhesion. It has been known that a small number of transmembrane proteins are associated with LRP. As this association is thought to be involved in the endocytosis and subsequent turnover of the membrane proteins, the mechanisms are insufficient to be understood. As LRP is a major binding

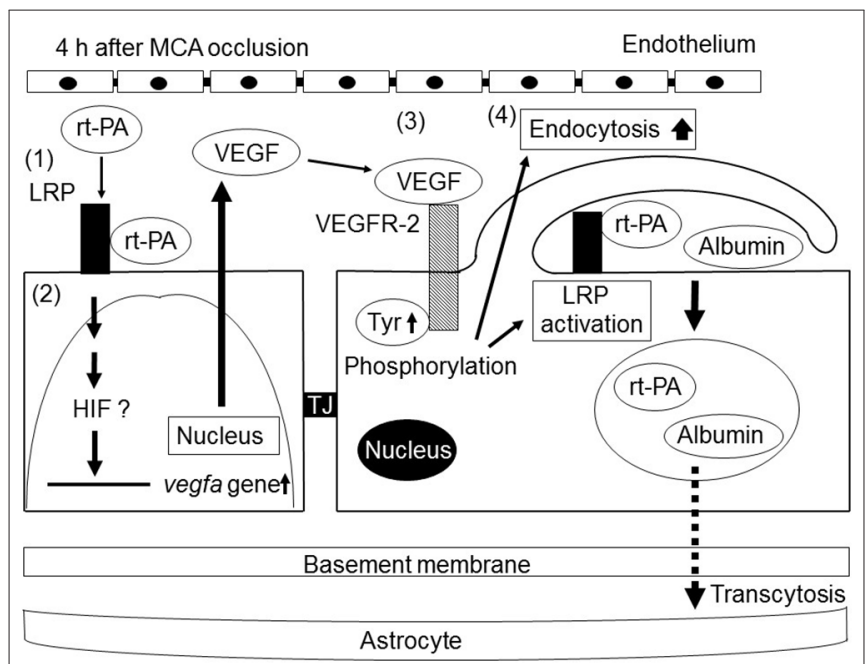

FIGURE 2 | Schematic of the mechanisms of the increase in BBB permeability by recombinant tissue-type plasminogen activator (rt-PA) treatment after ischemic stroke. rt-PA activates the low-density lipoprotein receptor-related protein (LRP), which is upregulated in endothelial cells (ECs) by ischemic stress (1). The activation of LRP induces the transcriptional upregulation of VEGF. Secreted VEGF binds to VEGFR-2 on the surface of ECs through an autocrine mechanism and induces its phosphorylation (3). The activation of VEGFR-2 leads to an increase in endocytosis and to the activation of LRP, resulting in enhanced BBB permeability by endocytosis and subsequent transcellular transport of proteins into cerebroparenchyma (4). Tyr, TJ. protein of $\mathrm{t}-\mathrm{PA}$, the administration of rt-PA may stimulate ECs and accelerate the endocytosis of plasma proteins via LRP and subsequent extravasation of proteins into the parenchyma.

\section{rt-PA, Plasmin, and the Substrate for Plasmin}

It is unclear whether the involvements of rt-PAs during ischemia, except for clot lysis, are associated with plasmin. Plasminogen is essentially present in both blood and the brain under most pathologic brain scenarios, especially together with rt-PA during its utilization in ischemic stroke. Plasminogen is exclusively localized in neurons of the cerebral cortex, hippocampus, hypothalamus, and the cerebellum in rodents (Tsirka et al., 1997; Basham and Seeds, 2001; Taniguchi et al., 2011). Hence, it is not likely to be obviously assumed that brain-derived plasminogen is activated at the $\mathrm{BBB}$ during stroke by endogenous t-PA. Blood-derived plasminogen may be more readily available by passing the $\mathrm{BBB}$, and it may be activated at the $\mathrm{BBB}$ under an ischemic condition. Therefore, brain-derived plasminogen in the neuronal pathology may not be completely associated with BBB permeability.

As previously described, plasmin can directly degrade basement membrane components or cause degradation of basement membrane components via the activation of MMPs (Lijnen, 2001), which possess similar basement membrane dismantling capabilities and damages the TJs (Jin et al., 2010). rt-PA treatment did not alter ICB associated with stroke in mice deficient in plasminogen and MMP-3, suggesting that plasmin may be required to activate MMP-3 by rt-PA in ECs during stroke (Suzuki et al., 2007).

Plasmin (and plasminogen) binds a wide array of cell-surface receptors or binding proteins and cleaves a variety of biologic substrates (Kwon et al., 2005; Miles and Parmer, 2013). As a result of this capacity, plasmin has been understood to play a role in many cellular responses, including cell migration, wound healing, tissue remodeling, apoptosis, cancer invasion, cancer metastasis, and inflammation and immunity, as extensively reviewed elsewhere (Kwon et al., 2005; Syrovets et al., 2012; Miles and Parmer, 2013). Together, these characters attribute a position to plasmin as a sound candidate that participates in the remodeling of cerebral blood vessels, especially during stroke, when the $\mathrm{BBB}$ weakens and blood components gain access to the $\mathrm{BBB}$. The direct intracortical injection of concentrated plasmin resulted in substantial lesion formation $6 \mathrm{~h}$ later, accompanied by the oxidation of proteins and DNA, degradation of occludin and collagen IV (basement membrane), and elevation of MMP-9, without the loss of ECs (Lukic-Panin et al., 2010). These findings indicate that plasmin can evidently influence TJ proteins and basement membrane. Therefore, it is likely that the administration of rt-PA accelerates the likelihood of a plasmindependent $\mathrm{BBB}$ alteration.

\section{CONCLUSIONS}

Delayed rt-PA treatment increases BBB permeability through a number of mechanisms. Although the mechanisms depend on 
the degree of cell damage after ischemia, rt-PA has the possibility of increasing $\mathrm{BBB}$ permeability without compromising $\mathrm{BBB}$ integrity and causing subsequent $\mathrm{BBB}$ breakdown. rt-PA increases BBB permeability via the induction of VEGF, which at least partially mediates the subsequent increase in endothelial endocytosis. Furthermore, an increase in $\mathrm{BBB}$ permeability by endocytosis is likely the first step in BBB breakdown by

\section{REFERENCES}

Abumiya, T., Yokota, C., Kuge, Y., and Minematsu, K. (2005). Aggravation of hemorrhagic transformation by early intraarterial infusion of low-dose vascular endothelial growth factor after transient focal cerebral ischemia in rats. Brain Res. 1049, 95-103. doi: 10.1016/j.brainres.2005.05.011

Argaw, A. T., Asp, L., Zhang, J., Navrazhina, K., Pham, T., Mariani, J. N., et al. (2012). Astrocyte-derived VEGF-A drives blood-brain barrier disruption in CNS inflammatory disease. J. Clin. Invest. 122, 2454-2468. doi: 10. 1172/JCI60842

Argaw, A. T., Gurfein, B. T., Zhang, Y., Zameer, A., and John, G. R. (2009). VEGFmediated disruption of endothelial CLN-5 promotes blood-brain barrier breakdown. Proc. Natl. Acad. Sci. U S A 106, 1977-1982. doi: 10.1073/pnas. 0808698106

Armulik, A., Genové, G., Mäe, M., Nisancioglu, M. H., Wallgard, E., Niaudet, C., et al. (2010). Pericytes regulate the blood-brain barrier. Nature 468, 557-561. doi: 10.1038/nature09522

Banai, S., Shweiki, D., Pinson, A., Chandra, M., Lazarovici, G., and Keshet, E. (1994). Upregulation of vascular endothelial growth factor expression induced by myocardial ischaemia: implications for coronary angiogenesis. Cardiovasc. Res. 28, 1176-1179. doi: 10.1093/cvr/28.8.1176

Basham, M. E., and Seeds, N. W. (2001). Plasminogen expression in the neonatal and adult mouse brain. J. Neurochem. 77, 318-325. doi: 10.1046/j.1471-4159. 2001.00239.x

Bell, R. D., Winkler, E. A., Sagare, A. P., Singh, I., LaRue, B., Deane, R., et al. (2010). Pericytes control key neurovascular functions and neuronal phenotype in the adult brain and during brain aging. Neuron 68, 409-427. doi: 10.1016/j.neuron. 2010.09.043

Benchenane, K., Berezowski, V., Ali, C., Fernández-Monreal, M., LópezAtalaya, J. P., Brillault, J., et al. (2005). Tissue-type plasminogen activator crosses the intact blood-brain barrier by low-density lipoprotein receptorrelated protein-mediated transcytosis. Circulation 111, 2241-2249. doi: 10. 1161/01.cir.0000163542.48611.a2

Brown, L. F., Yeo, K. T., Berse, B., Yeo, T. K., Senger, D. R., Dvorak, H. F., et al. (1992). Expression of vascular permeability factor (vascular endothelial growth factor) by epidermal keratinocytes during wound healing. J. Exp. Med. 176, 1375-1379. doi: 10.1084/jem.176.5.1375

Bu, G., Williams, S., Strickland, D. K., and Schwartz, A. L. (1992). Low density lipoprotein receptor-related protein/alpha 2 -macroglobulin receptor is an hepatic receptor for tissue-type plasminogen activator. Proc. Natl. Acad. Sci. U S A 89, 7427-7431. doi: 10.1073/pnas.89.16.7427

Castellanos, M., Leira, R., Serena, J., Pumar, J. M., Lizasoain, I., Castillo, J., et al. (2003). Plasma metalloproteinase- 9 concentration predicts hemorrhagic transformation in acute ischemic stroke. Stroke 34, 40-46. doi: 10.1161/01.STR. 0000046764.57344 .31

Chen, Z. L., and Strickland, S. (1997). Neuronal death in the hippocampus is promoted by plasmin-catalyzed degradation of laminin. Cell 91, 917-925. doi: 10.1016/s0092-8674(00)80483-3

Cheng, T., Petraglia, A. L., Li, Z., Thiyagarajan, M., Zhong, Z., Wu, Z., et al. (2006). Activated protein $C$ inhibits tissue plasminogen activator-induced brain hemorrhage. Nat. Med. 12, 1278-1285. doi: 10.1038/nm1498

Clark, W. M., Wissman, S., Albers, G. W., Jhamandas, J. H., Madden, K. P., and Hamilton, S. (1999). Recombinant tissue-type plasminogen activator (Alteplase) for ischemic stroke 3 to 5 hours after symptom onset. The ATLANTIS study: a randomized controlled trial. Alteplase thrombolysis for acute noninterventional therapy in ischemic stroke. JAMA 282, 2019-2026. doi: 10.1001/jama.282.21.2019 delayed rt-PA treatment combined with ischemic stroke because various plasma proteins in the bloodstream are taken up by the parenchyma across the compromised BBB.

\section{AUTHOR CONTRIBUTIONS}

YS and NN: planning and writing the review. KU: supervisor.

Daneman, R., Zhou, L., Kebede, A. A., and Barres, B. A. (2010). Pericytes are required for blood- brain barrier integrity during embryogenesis. Nature 468, 562-566. doi: 10.1038/nature09513

Dedieu, S., Langlois, B., Devy, J., Sid, B., Henriet, P., Sartelet, H., et al. (2008). LRP-1 silencing prevents malignant cell invasion despite increased pericellular proteolytic activities. Mol. Cell. Biol. 28, 2980-2995. doi: 10.1128/MCB. 02238-07

Dellian, M., Witwer, B. P., Salehi, H. A., Yuan, F., and Jain, R. K. (1996). Quantitation and physiological characterization of angiogenic vessels in mice: effect of basic fibroblast growth factor, vascular endothelial growth factor/vascular permeability factor and host microenvironment. Am. J. Pathol. 149, 59-71.

Duan, P., and Ni, C. (2014). t-PA stimulates VEGF expression in endothelial cells via ERK2/p38 signaling pathways. Pharmazie 69, 70-75. doi: 10.1691/ph. 2014.3655

Durukan, A., Marinkovic, I., Strbian, D., Pitkonen, M., Pedrono, E., Soinne, L., et al. (2009). Post-ischemic blood-brain barrier leakage in rats: one-week follow-up by MRI. Brain Res. 1280, 158-165. doi: 10.1016/j.brainres.2009. 05.025

Dvorak, H. F., Brown, L. F., Detmar, M., and Dvorak, A. M. (1995). Vascular permeability factor/vascular endothelial growth factor, microvascular hyperpermeability and angiogenesis. Am. J. Pathol. 146, 1029-1039.

Ema, M., Taya, S., Yokotani, N., Sogawa, K., Matsuda, Y., and Fujii-Kuriyama, Y. (1997). A novel bHLH-PAS factor with close sequence similarity to hypoxiainducible factor $1 \alpha$ regulates the VEGF expression and is potentially involved in lung and vascular development. Proc. Natl. Acad. Sci. U S A 94, 4273-4278. doi: $10.1073 /$ pnas.94.9.4273

Etique, N., Verzeaux, L., Dedieu, S., and Emonard, H. (2013). LRP-1: a checkpoint for the extracellular matrix proteolysis. BioMed Res. Int. 2013:152163. doi: 10. $1155 / 2013 / 152163$

Fischer, S., Gerriets, T., Wessels, C., Walberer, M., Kostin, S., Stolz, E., et al. (2007). Preissner. Extracellular RNAmediates endothelial-cell permeability via vascular endothelial growth factor. Blood 110, 2457-2465. doi: 10.1182/blood-2006-08040691

Fischer, S., Wobben, M., Marti, H. H., Renz, D., and Schaper, W. (2002). Hypoxiainduced hyperpermeability in brain microvessel endothelial cells involves VEGF-mediated changes in the expression of zonula occludens-1. Microvasc. Res. 63, 70-80. doi: 10.1006/mvre.2001.2367

Forsythe, J. A., Jiang, B. H., Iyer, N. V., Agani, F., Leung, S. W., Koos, R. D., et al. (1996). Activation of vascular endothelial growth factor gene transcription by hypoxia-inducible factor 1. Mol. Cell. Biol. 16, 4604-4613. doi: 10.1128/mcb.16. 9.4604

Hacke, W., Kaste, M., Bluhmki, E., Brozman, M., Dávalos, A., Guidetti, D., et al. (2008). ECASS Investigators. Thrombolysis with alteplase 3 to 4.5 hours after acute ischemic stroke. N. Engl. J. Med. 359, 1317-1329. doi: 10. 1056/NEJMoa0804656

Hawkins, B. T., and Davis, T. P. (2005). The blood-brain barrier/neurovascular unit in health and disease. Pharmacol. Rev. 57, 173-185. doi: 10.1124/pr. 57.2 .4

Hayashi, T., Abe, K., Suzuki, H., and Itoyama, Y. (1997). Rapid induction of vascular endothelial growth factor gene expression after transient middle cerebral artery occlusion in rats. Stroke 28, 2039-2044. doi: 10.1161/01.str.28. 10.2039

Herz, J., Hamann, U., Rogne, S., Myklebost, O., Gausepohl, H., and Stanley, K. K. (1988). Surface location and high affinity for calcium of a 500-kd liver membrane protein closely related to the LDL-receptor suggest a physiological role as lipoprotein receptor. EMBO J. 7, 4119-4127. 
Hirase, T., Staddon, J. M., Saitou, M., Ando-Akatsuka, Y., Itoh, M., Furuse, M., et al. (1997). Occludin as a possible determinant of tight junction permeability in endothelial cells. J. Cell Sci. 110, 1603-1613.

Horowitz, A., and Seerapu, H. R. (2012). Regulation of VEGF signaling by membrane traffic. Cell. Signal. 24, 1810-1820. doi: 10.1016/j.cellsig.2012.05.007

Horstmann, S., Kalb, P., Koziol, J., Gardner, H., and Wagner, S. (2003). Profiles of matrix metalloproteinases, their inhibitors and laminin in stroke patients: influence of different therapies. Stroke 34, 2165-2170. doi: 10.1161/01. STR.0000088062.86084.F2

Ikeda, E., Achen, M. G., Breier, G., and Risau, W. (1995). Hypoxia-induced transcriptional activation and increased mRNA stability of vascular endothelial growth factor (VEGF) in C6 glioma cells. J. Biol. Chem. 270, 19761-19766. doi: $10.1074 /$ jbc.270.34.19761

Ishiguro, M., Mishiro, K., Fujiwara, Y., Chen, H., Izuta, H., Tsuruma, K., et al. (2010). Phosphodiesterase-III inhibitor prevents hemorrhagic transformation induced by focal cerebral ischemia in mice treated with tPA. PLoS One 5:e15178. doi: 10.1371/journal.pone.0015178

Jickling, G. C., Liu, D., Stamova, B., Ander, B. P., Zhan, X., Lu, A., et al. (2014). Hemorrhagic transformation after ischemic stroke in animals and humans. J. Cereb. Blood Flow. Metab. 34, 185-199. doi: 10.1038/jcbfm. 2013.203

Jin, R., Yang, G., and Li, G. (2010). Molecular insights and therapeutic targets for blood-brain barrier disruption in ischemic stroke: critical role of matrix metalloproteinases and tissue-type plasminogen activator. Neurobiol. Dis. 38, 376-385. doi: 10.1016/j.nbd.2010.03.008

Kahles, T., and Brandes, R. P. (2012). NADPH oxidases as therapeutic targets in ischemic stroke. Cell. Mol. Life Sci. 69, 2345-2363. doi: 10.1007/s00018-0121011-8

Kanazawa, M., Igarashi, H., Kawamura, K., Takahashi, T., Kakita, A., Takahashi, H., et al. (2011). Inhibition of VEGF signaling pathway attenuates hemorrhage after tPA treatment. J. Cereb. Blood Flow Metab. 31, 1461-1474. doi: $10.1038 /$ jcbfm.2011.9

Kappel, A., Rönicke, V., Damert, A., Flamme, I., Risau, W., and Breier, G. (1999). Identification of vascular endothelial growth factor (VEGF) receptor-2 (Flk-1) promoter/enhancer sequences sufficient for angioblast and endothelial cellspecific transcription in transgenic mice. Blood 93, 4284-4292.

Kassner, A., Roberts, T. P., Moran, B., Silver, F. L., and Mikulis, D. J. (2009). Recombinant tissue plasminogen activator increases blood-brain barrier disruption in acute ischemic stroke: an MR imaging permeability study. Am. J. Neuroradiol. 30, 1864-1869. doi: 10.3174/ajnr.a1774

Kastrup, A., Gröschel, K., Ringer, T. M., Redecker, C., Cordesmeyer, R., Witte, O. W., et al. (2008). Early disruption of the blood-brain barrier after thrombolytic therapy predicts hemorrhage in patients with acute stroke. Stroke 39, 2385-2387. doi: 10.1161/STROKEAHA.107.505420

Kaur, J., Zhao, Z., Klein, G. M., Lo, E. H., and Buchan, A. M. (2004). The neurotoxicity of tissue plasminogen activator? J. Cereb. Blood Flow Metab. 24, 945-963. doi: 10.1097/01.wcb.0000137868.50767.e8

Kazmierski, R., Michalak, S., Wencel-Warot, A., and Nowinski, W. L. (2012). Serum tight-junction proteins predict hemorrhagic transformation in ischemic stroke patients. Neurology 79, 1677-1685. doi: 10.1212/WNL. 0b013e31826e9a83

Kevil, C. G., Oshima, T., Alexander, B., Coe, L. L., and Alexander, J. S. (2000). $\mathrm{H}_{2} \mathrm{O}_{2}$-mediated permeability: role of MAPK and occludin. Am. J. Physiol. Cell Physiol. 279, C21-C30.

Kovács, Z., Ikezaki, K., Samoto, K., Inamura, T., and Fukui, M. (1996). VEGF and flt. Expression time kinetics in rat brain infarct. Stroke 27, 1865-1872; discussion 1872-1873. doi: 10.1161/01.str.27.10.1865

Krueger, M., Härtig, W., Reichenbach, A., Bechmann, I., and Michalski, D. (2013). Blood-brain barrier breakdown after embolic stroke in rats occurs without ultrastructural evidence for disrupting tight junctions. PLoS One 8:e56419. doi: 10.1371/journal.pone.0056419

Kwon, M., MacLeod, T. J., Zhang, Y., and Waisman, D. M. (2005). S100A10, annexin A2 and annexin a2 heterotetramer as candidate plasminogen receptors. Front. Biosci. 10, 300-325. doi: 10.2741/1529

Lee, H. S., Namkoong, K., Kim, D. H., Kim, K. J., Cheong, Y. H., Kim, S. S., et al. (2004). Hydrogen-peroxide-induced alterations of tight junction proteins in bovine brain microvascular endothelial cells. Microvasc. Res. 68, 231-238. doi: 10.1016/j.mvr.2004.07.005
Levy, A. P., Levy, N. S., Wegner, S., and Goldberg, M. A. (1995). Transcriptional regulation of the rat vascular endothelial growth factor gene by hypoxia. J. Biol. Chem. 270, 13333-13340. doi: 10.1074/jbc.270.22.13333

Lijnen, H. R. (2001). Plasmin and matrix metalloproteinases in vascular remodeling. Thromb. Haemost. 86, 324-333.

Lijnen, H. R., and Collen, D. (1987). Tissue-type plasminogen activator. Ann. Biol. Clin. (Paris) 45, 198-201.

Liu, Y., Cox, S. R., Morita, T., and Kourembanas, S. (1995). Hypoxia regulates vascular endothelial growth factor gene expression in endothelial cells. Identification of a $5^{\prime}$ enhancer. Circ. Res. 77, 638-643. doi: 10.1161/01.res.77. 3.638

Lochhead, J. J., McCaffrey, G., Quigley, C. E., Finch, J., DeMarco, K. M., Nametz, N., et al. (2010). Oxidative stress increases blood-brain barrier permeability and induces alterations in occludin during hypoxiareoxygenation. J. Cereb. Blood Flow Metab. 30, 1625-1636. doi: 10.1038/jcbfm. 2010.29

López-Atalaya, J. P., Roussel, B. D., Ali, C., Maubert, E., Petersen, K. U., Berezowski, V. R., et al. (2007). Recombinant Desmodus rotundus salivary plasminogen activator crosses the blood-brain barrier through a low-density lipoprotein receptor-related protein-dependent mechanism without exerting neurotoxic effects. Stroke 38, 1036-1043. doi: 10.1161/01.str.0000258100. 04923.84

Lukic-Panin, V., Deguchi, K., Yamashita, T., Shang, J., Zhang, X., Tian, F., et al. (2010). Free radical scavenger edaravone administration protects against tissue plasminogen activator induced oxidative stress and blood brain barrier damage. Curr. Neurovasc. Res. 7, 319-329. doi: 10.2174/1567202107931 80747

Mackay, A. R., Corbitt, R. H., Hartzler, J. L., and Thorgeirsson, U. P. (1990). Basement membrane type IV collagen degradation: evidence for the involvement of a proteolytic cascade independent of metalloproteinases. Cancer Res. 50, 5997-6001.

Madara, J. L., Barenberg, D., and Carlson, S. (1986). Effects of cytochalasin D on occluding junctions of intestinal absorptive cells: further evidence that the cytoskeleton may influence paracellular permeability and junctional charge selectivity. J. Cell Biol. 102, 2125-2136. doi: 10.1083/jcb.102.6.2125

Marchina, E., and Barlati, S. (1996). Degradation of human plasma and extracellular matrix fibronectin by tissue type plasminogen activator and urokinase. Int. J. Biochem. Cell Biol. 28, 1141-1150. doi: 10.1016/13572725(96)00055-6

Mark, K. S., and Davis, T. P. (2002). Cerebral microvascular changes in permeability and tight junctions induced by hypoxia-reoxygenation. Am. J. Physiol. Heart Circ. Physiol. 282, H1485-H1494. doi: 10.1152/ajpheart.00645. 2001

Marti, H. J., Bernaudin, M., Bellail, A., Schoch, H., Euler, M., Petit, E., et al. (2000). Hypoxia-induced vascular endothelial growth factor expression precedes neovascularization after cerebral ischemia. Am. J. Pathol. 156, 965-976. doi: 10. 1016/s0002-9440(10)64964-4

Marti, H. H., and Risau, W. (1998). Systemic hypoxia changes the organ-specific distribution of vascular endothelial growth factor and its receptors. Proc. Natl. Acad. Sci. U S A 95, 15809-15814. doi: 10.1073/pnas.95.26.15809

Martìn-Padura, I., Lostaglio, S., Schneemann, M., Williams, L., Romano, M., Fruscella, P., et al. (1998). Junctional adhesion molecule, a novel member of the immunoglobulin superfamily that distributes at intercellular junctions and modulates monocyte transmigration. J. Cell Biol. 142, 117-127. doi: 10. 1083/jcb.142.1.117

Miles, L. A., and Parmer, R. J. (2013). Plasminogen receptors: the first quarter century. Semin. Thromb. Hemost. 39, 329-337. doi: 10.1055/s-0033-1334483

Mishiro, K., Ishiguro, M., Suzuki, Y., Tsuruma, K., Shimazawa, M., and Hara, H. (2012). A broad-spectrum matrix metalloproteinase inhibitor prevents hemorrhagic complications induced by tissue plasminogen activator in mice. Neuroscience 205, 39-48. doi: 10.1016/j.neuroscience.2011.12.042

Monacci, W. T., Merrill, M. J., and Oldfield, E. H. (1993). Expression of vascular permeability factor/vascular endothelial growth factor in normal rat tissues. Am. J. Physiol. 264, C995-C1002.

Nagai, N., De Mol, M., Lijnen, H. R., Carmeliet, P., and Collen, D. (1999). Role of plasminogen system components in focal cerebral ischemic infarction: a gene targeting and gene transfer study in mice. Circulation 99, 2440-2444. doi: 10. 1161/01.cir.99.18.2440 
Nagase, H., and Woessner, J. F., Jr. (1999). Matrix metalloproteinases. J. Biol. Chem. 274, 21491-21494. doi: 10.1074/jbc.274.31.21491

Nakayama, M., and Berger, P. (2013). Coordination of VEGF receptor trafficking and signaling by coreceptors. Exp. Cell Res. 319, 1340-1347. doi: 10.1016/j. yexcr.2013.03.008

Nicole, O., Docagne, F., Ali, C., Margaill, I., Carmeliet, P., MacKenzie, E. T., et al. (2001). The proteolytic activity of tissue-plasminogen activator enhances NMDA receptor-mediated signaling. Nat. Med. 7, 59-64. doi: 10.1038/83358

Niego, B., and Medcalf, R. L. (2014). Plasmin-dependent modulation of the blood-brain barrier: a major consideration during tPA-induced thrombolysis? J. Cereb. Blood Flow Metab. 34, 1283-1296. doi: 10.1038/jcbfm.2014.99

Nour, M., Scalzo, F., and Liebeskind, D. S. (2013). Ischemia-reperfusion injury in stroke. Interv. Neurol. 1, 185-199. doi: 10.1159/000353125

Obermeier, B., Daneman, R., and Ransohoff, R. M. (2013). Development, maintenance and disruption of the blood-brain barrier. Nat. Med. 19, 1584-1596. doi: 10.1038/nm.3407

Ohki, M., Ohki, Y., Ishihara, M., Nishida, C., Tashiro, Y., Akiyama, H., et al. (2010). Tissue type plasminogen activator regulates myeloid-cell dependent neoangiogenesis during tissue regeneration. Blood 115, 4302-4312. doi: 10. 1182/blood-2009-08-236851

Pedram, A., Razandi, M., and Levin, E. R. (2002). Deciphering vascular endothelial cell growth factor/vascular permeability factor signaling to vascular permeability. J. Biol. Chem. 277, 44385-44398. doi: 10.1074/jbc.m202391200

Pun, P. B., Lu, J., and Moochhala, S. (2009). Involvement of ROS in BBB dysfunction. Free Radic. Res. 43, 348-364. doi: 10.1080/10715760902751902

Ramirez, S. H., Potula, R., Fan, S., Eidem, T., Papugani, A., Reichenbach, N., et al. (2009). Methamphetamine disrupts blood-brain barrier function by induction of oxidative stress in brain endothelial cells. J. Cereb. Blood Flow Metab. 29, 1933-1945. doi: $10.1038 / \mathrm{jcbfm} .2009 .112$

Reese, T. S., and Karnovsky, M. J. (1967). Fine structural localization of a bloodbrain barrier to exogenous peroxidase. J. Cell Biol. 34, 207-217. doi: 10. 1083/jcb.34.1.207

Reuter, B., Rodemer, C., Grudzenski, S., Couraud, P. O., Weksler, B., Romero, I. A., et al. (2013). Temporal profile of matrix metalloproteinases and their inhibitors in a human endothelial cell culture model of cerebral ischemia. Cerebrovasc. Dis. 35, 514-520. doi: 10.1159/000350731

Rogove, A. D., and Tsirka, S. E. (1998). Neurotoxic responses by microglia elicited by excitotoxic injury in the mouse hippocampus. Curr. Biol. 8, 19-25. doi: 10. 1016/s0960-9822(98)70016-8

Rosell, A., Cuadrado, E., Ortega-Aznar, A., Hernández-Guillamon, M., Lo, E. H., and Montaner, J. (2008). MMP-9-positive neutrophil infiltration is associated to blood-brain barrier breakdown and basal lamina type IV collagen degradation during hemorrhagic transformation after human ischemic stroke. Stroke 39, 1121-1126. doi: 10.1161/STROKEAHA.107.500868

Rosenberg, G. A., Estrada, E. Y., Dencoff, J. E., and Stetler-Stevenson, W. G. (1995). Tumor necrosis factor-alpha-induced gelatinase B causes delayed opening of the blood-brain barrier: an expanded therapeutic window. Brain Res. 703, 151-155. doi: 10.1016/0006-8993(95)01089-0

Roux, F., and Couraud, P. O. (2005). Rat brain endothelial cell lines for the study of blood-brain barrier permeability and transport functions. Cell Mol. Neurobiol. 25, 41-58. doi: 10.1007/s10571-004-1376-9

Saunders, N. R., Liddelow, S. A., and Dziegielewska, K. M. (2012). Barrier mechanisms in the developing brain. Front. Pharmacol. 3:46. doi: 10. 3389/fphar.2012.00046

Sharp, F. R., and Bernaudin, M. (2004). HIF1 and oxygen sensing in the brain. Nat. Rev. Neurosci. 5, 437-448. doi: 10.1038/nrn1408

Shi, H. (2009). Hypoxia inducible factor 1 as a therapeutic target in ischemic stroke. Curr. Med. Chem. 16, 4593-4600. doi: 10.2174/092986709789760779

Siegenthaler, J. A., Sohet, F., and Daneman, R. (2013). 'Sealing off the CNS': cellular and molecular regulation of blood-brain barriergenesis. Curr. Opin. Neurobiol. 13, 1057-1064. doi: 10.1016/j.conb.2013.06.006

Stein, I., Itin, A., Einat, P., Skaliter, R., Grossman, Z., and Keshet, E. (1998). Translation of vascular endothelial growth factor mRNA by internal ribosome entry: implications for translation under hypoxia. Mol. Cell. Biol. 18, 3112-3119. doi: $10.1128 / \mathrm{mcb} .18 .6 .3112$

Su, E. J., Fredriksson, L., Geyer, M., Folestad, E., Cale, J., Andrae, J., et al. (2008). Activation of PDGF-CC by tissue plasminogen activator impairs bloodbrain barrier integrity during ischemic stroke. Nat. Med. 14, 731-737. doi: 10. 1038/nm1787
Suzuki, Y., Nagai, N., Umemura, K., Collen, D., and Lijnen, H. R. (2007). Stromelysin-1 (MMP-3) is critical for intracranial bleeding after t-PA treatment of stroke in mice. J. Thromb. Haemost. 5, 1732-1739. doi: 10.1111/j.1538-7836. 2007.02628.x

Suzuki, Y., Nagai, N., Yamakawa, K., Kawakami, J., Lijnen, H. R., and Umemura, K. (2009). Tissue-type plasminogen activator (t-PA) induces stromelysin-1 (MMP-3) in endothelial cells through activation of lipoprotein receptor-related protein. Blood 114, 3352-3358. doi: 10.1182/blood-2009-02203919

Suzuki, Y., Nagai, N., Yamakawa, K., Muranaka, Y., Hokamura, K., and Umemura, K. (2015). Recombinant tissue-type plasminogen activator transiently enhances blood-brain barrier permeability during cerebral ischemia through vascular endothelial growth factor-mediated endothelial endocytosis in mice. J. Cereb. Blood Flow Metab. 35, 2021-2031. doi: 10. $1038 /$ jcbfm.2015.167

Syrovets, T., Lunov, O., and Simmet, T. (2012). Plasmin as a proinflammatory cell activator. J. Leukoc. Biol. 92, 509-519. doi: 10.1189/jlb.0212056

Tang, N., Wang, L., Esko, J., Giordano, F. J., Huang, Y., Gerber, H. P., et al. (2004). Loss of HIF-1alpha in endothelial cells disrupts a hypoxia-driven VEGF autocrine loop necessary for tumorigenesis. Cancer Cell. 6, 485-495. doi: 10. 1016/j.ccr.2004.09.026

Taniguchi, Y., Inoue, N., Morita, S., Nikaido, Y., Nakashima, T., Nagai, N., et al. (2011). Localization of plasminogen in mouse hippocampus, cerebral cortex and hypothalamus. Cell Tissue Res. 343, 303-317. doi: 10.1007/s00441-010$1110-5$

The National Institute of Neurological Disorders and Stroke rt-PA Stroke Study Group. (1995). Tissue plasminogen activator for acute ischemic stroke. N. Engl. J. Med. 333, 1581-1587. doi: 10.1056/NEJM199512143332401

Tsirka, S. E., Rogove, A. D., Bugge, T. H., Degen, J. L., and Strickland, S. (1997). An extracellular proteolytic cascade promotes neuronal degeneration in the mouse hippocampus. J. Neurosci. 17, 543-552.

Turner, R. J., and Vink, R. (2012). Combined tissue plasminogen activator and an NK1 tachykinin receptor antagonist: an effective treatment for reperfusion injury following acute ischemic stroke in rats. Neuroscience 220, 1-10. doi: 10. 1016/j.neuroscience.2012.06.047

Wachtel, M., Frei, K., Ehler, E., Fontana, A., Winterhalter, K., and Gloor, S. M. (1999). Occludin proteolysis and increased permeability in endothelial cells through tyrosine phosphatase inhibition. J. Cell Sci. 112, 4347-4356.

Wang, X., Lee, S. R., Arai, K., Tsuji, K., Rebeck, G. W., and Lo, E. H. (2003). Lipoprotein receptor-mediated induction of matrix metalloproteinase by tissue plasminogen activator. Nat. Med. 9, 1313-1317. doi: 10.1038/nm926

Wang, C. X., and Shuaib, A. (2007). Critical role of microvasculature basal lamina in ischemic brain injury. Prog. Neurobiol. 83, 140-148. doi: 10.1016/j. pneurobio.2007.07.006

Wang, Y. F., Tsirka, S. E., Strickland, S., Stieg, P. E., Soriano, S. G., and Lipton, S. A. (1998). Tissue plasminogen activator (tPA) increases neuronal damage after focal cerebral ischemia in wild-type and tPA-deficient mice. Nat. Med. 4, 228-231. doi: 10.1038/nm0298-228

Wenger, R. H., and Gassmann, M. (1997). Oxygen(es) and the hypoxia-inducible factor-1. Biol. Chem. 378, 609-616.

Witt, K. A., Mark, K. S., Hom, S., and Davis, T. P. (2003). Effects of hypoxiareoxygenation on rat blood-brain barrier permeability and tight junctional protein expression. Am. J. Physiol. Heart Circ. Physiol. 285, H2820-H2831. doi: 10.1152/ajpheart.00589.2003

Won, S., Lee, J. H., Wali, B., Stein, D. G., and Sayeed, I. (2014). Progesterone attenuates hemorrhagic transformation after delayed tPA treatment in an experimental model of stroke in rats: involvement of the VEGF-MMP pathway. J. Cereb. Blood Flow Metab. 34, 72-80. doi: 10.1038/jcbfm.2013.163

Wu, F., Wu, J., Nicholson, A. D., Echeverry, R., Haile, W. B., Catano, M., et al. (2012). Tissue-type plasminogen activator regulates the neuronal uptake of glucose in the ischemic brain. J. Neurosci. 32, 9848-9858. doi: 10 . 1523/jneurosci.1241-12.2012

Yamashita, T., Kamiya, T., Deguchi, K., Inaba, T., Zhang, H., Shang, J., et al. (2009). Dissociation and protection of the neurovascular unit after thrombolysis and reperfusion in ischemic rat brain. J. Cereb. Blood Flow Metab. 29, 715-725. doi: $10.1038 / j \mathrm{cbfm} .2008 .164$

Yang, Y., Estrada, E. Y., Thompson, J. F., Liu, W., and Rosenberg, G. A. (2007). Matrix metalloproteinase-mediated disruption of tight junction proteins in cerebral vessels is reversed by synthetic matrix metalloproteinase inhibitor 
in focal ischemia in rat. J. Cereb. Blood Flow Metab. 27, 697-709. doi: 10. 1038/sj.jcbfm.9600375

Yang, Y., Thompson, J. F., Taheri, S., Salayandia, V. M., McAvoy, T. A., Hill, J. W., et al. (2013). Early inhibition of MMP activity in ischemic rat brain promotes expression of tight junction proteins and angiogenesis during recovery. J. Cereb. Blood Flow Metab. 33, 1104-1114. doi: 10.1038/jcbfm. 2013.56

Ye, J., Tsukamoto, T., Sun, A., and Nigam, S. K. (1999). A role for intracellular calcium in tight junction reassembly after ATP depletion-repletion. Am. J. Physiol. 277, F524-F532.

Yepes, M., Sandkvist, M., Moore, E. G., Bugge, T. H., Strickland, D. K., and Lawrence, D. A. (2003). Tissue-type plasminogen activator induces opening of the blood-brain barrier via the LDL receptor-related protein. J. Clin. Invest. 112, 1533-1540. doi: 10.1172/jci200319212

Zechariah, A., Elali, A., Doeppner, T. R., Jin, F., Hasan, M. R., Helfrich, I., et al. (2013). Vascular endothelial growth factor promotes pericyte coverage of brain capillaries, improves cerebral blood flow during subsequent focal cerebral ischemia and preserves the metabolic penumbra. Stroke 44, 1690-1697. doi: 10. 1161/STROKEAHA.111.000240

Zhang, Z. G., Zhang, L., Jiang, Q., Zhang, R., Davies, K., Powers, C., et al. (2000). VEGF enhances angiogenesis and promotes blood-brain barrier leakage in the ischemic brain. J. Clin. Invest. 106, 829-838. doi: 10.1172/ jci9369

Conflict of Interest Statement: The authors declare that the research was conducted in the absence of any commercial or financial relationships that could be construed as a potential conflict of interest.

Copyright (c) 2016 Suzuki, Nagai and Umemura. This is an open-access article distributed under the terms of the Creative Commons Attribution License (CC BY). The use, distribution and reproduction in other forums is permitted, provided the original author(s) or licensor are credited and that the original publication in this journal is cited, in accordance with accepted academic practice. No use, distribution or reproduction is permitted which does not comply with these terms. 